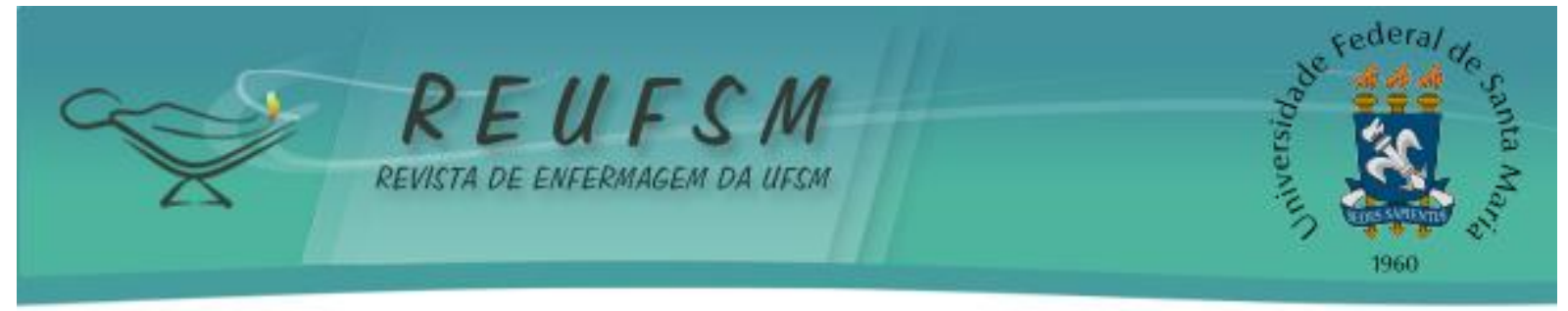

ARTIGO ORIGINAL

\title{
PERFIL DAS MULHERES EM TRATAMENTO QUIMIOTERÁPICO EM UM MUNICÍPIO NO SUL DO BRASIL*
}

\section{PROFILE OF WOMEN UNDERGOING CHEMOTHERAPY IN A CITY IN SOUTHERN BRAZIL PERFIL DE LAS MUJERES EN TRATAMIENTO QUIMIOTERÁPICO EN UNA CIUDAD DEL SUR DE BRASIL}

\author{
Aline da Costa Viegas ${ }^{1}$ \\ Eda Schwartz ${ }^{2}$ \\ Juliana Graciela Vestena Zillmer ${ }^{3}$ \\ Rosani Manfrin Muniz ${ }^{4}$ \\ Bianca Pozza dos Santos ${ }^{5}$ \\ Daiane Lopes Leal Borba ${ }^{6}$ \\ Aline Machado Feijó ${ }^{7}$
}

Doi: $10.5902 / 2179769210530$

RESUMO: Objetivo: descrever o perfil das mulheres em tratamento quimioterápico em um serviço de oncologia de um hospital de ensino em Pelotas, Rio Grande do Sul, Brasil. Método: estudo descritivo, com abordagem quantitativa, realizado de março a junho de 2010. A amostra constituiu-se de 117 mulheres. A análise dos dados foi frequencial. Resultados: as características predominantes foram: idosas, cor branca, sem companheiro, que estudaram entre cinco a oito anos, com alguma crença religiosa, aposentadas, com baixa renda e procedentes do município em que se originou o estudo. A comorbidade de maior frequência foi a hipertensão, quanto aos fatores de risco modificáveis, foram o uso do tabaco e a ingestão de chimarrão em temperatura elevada. Em relação aos tipos de câncer, o de mama foi o mais prevalente. Conclusão: este estudo permitiu conhecer as características sociodemográficas e econômicas das mulheres com câncer, podendo dessa forma, direcionar ações específicas para esta população.

\footnotetext{
*Manuscrito elaborado a partir da monografia: Caracterização das mulheres em tratamento quimioterápico de um serviço de oncologia situado em um município no sul do Brasil. Apresentado em 2011 à Faculdade de Enfermagem da Universidade Federal de Pelotas, tendo como orientadora Enf ${ }^{a}$. Prof ${ }^{a}$. Dr ${ }^{a}$. Eda Schwartz e coorientadora Enf ${ }^{a}$. Me. Juliana Graciela Vestena Zillmer.

${ }^{1}$ Enfermeira. Mestre em Ciências. Doutoranda pelo Programa de Pós-Graduação em Enfermagem da Faculdade de Enfermagem da Universidade Federal de Pelotas (UFPel). Membro do Núcleo de Condições Crônicas e suas Interfaces (NUCCRIN). Pelotas, Rio Grande do Sul (RS), Brasil. E-mail: alinecviegas@hotmail.com

${ }^{2}$ Enfermeira. Pós-Doutora em Enfermagem. Docente da Faculdade de Enfermagem da UFPel. Pesquisadora do NUCCRIN e vice-líder do Núcleo de Pesquisa em Saúde Rural e Sustentabilidade. Pelotas, RS, Brasil. E-mail: eschwartz@terra.com.br

${ }^{3}$ Enfermeira. Mestre em Ciências. Doutoranda pelo Programa de Pós-Graduação em Enfermagem da Universidade Federal de Santa Catarina (UFSC). Docente da Faculdade de Enfermagem da UFPel. Membro do NUCCRIN e do Núcleo de Estudos e Assistência em Enfermagem e Saúde às Pessoas com Doenças Crônicas (NUCRON). Pelotas, RS, Brasil. E-mail: juzillmer@gmail.com

${ }^{4}$ Enfermeira. Doutora em Enfermagem. Docente da Faculdade de Enfermagem da UFPel. Líder e Pesquisadora do NUCCRIN. Pelotas, RS, Brasil. E-mail: romaniz@terra.com.br

${ }^{5}$ Enfermeira. Mestre em Ciências. Doutoranda pelo Programa de Pós-Graduação em Enfermagem da UFPel. Enfermeira contratada pela Prefeitura Municipal de Pelotas. Membro do NUCCRIN e do Núcleo de Pesquisa em Saúde Rural e Sustentabilidade. Pelotas, RS, Brasil. E-mail: bi.santos@bol.com.br

${ }^{6}$ Enfermeira. Enfermeira Assistencial do Hospital Universitário São Francisco de Paula. Pelotas, RS, Brasil. Email: daianelleal@yahoo.com.br

${ }^{7}$ Enfermeira. Mestre em Ciências. Doutoranda pelo Programa de Pós-Graduação em Enfermagem da UFPel. Enfermeira do Hemocentro Regional de Pelotas (HEMOPEL). Membro do NUCCRIN e do Núcleo de Pesquisa em Saúde Rural e Sustentabilidade. Pelotas, RS, Brasil. E-mail: aline_feijo@yahoo.com.br
} 


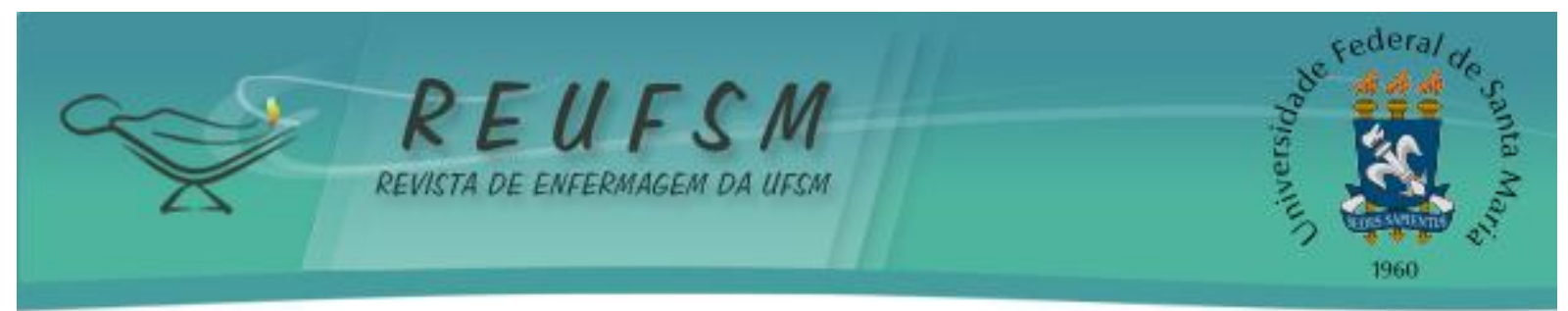

Descritores: Neoplasias; Quimioterapia; Mulheres; Enfermagem; Perfil de saúde.

ABSTRACT: Aim: to describe the profile of women undergoing chemotherapy in an oncology service of a teaching hospital in Pelotas, Rio Grande do Sul, Brazil. Method: descriptive study with a quantitative approach, conducted from March to June in 2010. The participants were 117 women. Data analysis was frequencial. Results: the predominant features were: elderly women, white, unmarried, who studied for five to eight years, with some religious belief, retired, with low income, and from the city where the study originated. Hypertension was the most frequent comorbidity. The use of tobacco and the ingestion of mate in high temperature were the modifiable risk factors. In regards to the types of cancer, breast cancer was the most prevalent. Conclusion: this study helped to identify the sociodemographic and economic characteristics of women with cancer, enabling specific actions targeting this population.

Descriptors: Neoplasms; Drug therapy; Women; Nursing; Health profile.

RESUMEN: Objetivo: describir el perfil de las mujeres en tratamiento quimioterápico en un servicio de oncología de un hospital universitario en Pelotas, (Rio Grande do Sul, Brasil). Método: estudio descriptivo, con enfoque cuantitativo, realizado entre marzo y junio de 2010. La muestra fue constituída por 117 mujeres. El análisis de datos fue frecuencial. Resultados: las características predominantes fueron: ancianas, color blanco, sin compañero, estudiaron entre cinco y ocho años, con alguna creencia religiosa, jubiladas, con renta baja y procedentes de la ciudad en que se originó el estudio. El trastorno comórbido más frecuente fue la hipertensión, los factores de riesgo modificables fueron el uso del tabaco y la ingestión de mate en temperatura elevada. De los tipos de cáncer, el de mama fue el más prevalente. Conclusión: este estudio permitió conocer las características sociodemográficas y económicas de las mujeres con cáncer, pudiendo direccionar acciones específicas para esta población.

Descriptores: Neoplasias; Quimioterapia; Mujeres; Enfermería; Perfil de salud.

\section{INTRODUÇÃO}

Mundialmente, o câncer consiste em uma das doenças que interfere no aumento das taxas de mortalidade, atingindo mais de 25 milhões de pessoas. Segundo estimativa da Organização Mundial da Saúde, as neoplasias foram a principal causa de morte, em 7,6 milhões de pessoas no ano de 2008. ${ }^{1}$

As estimativas para os anos de 2012 e 2013 sugerem a ocorrência de 518.510 casos novos de câncer no Brasil. Os tipos mais incidentes apontados para as mulheres foram os tumores de pele (não melanoma), mama, colo do útero, cólon e reto e glândula tireóide. ${ }^{2}$

Diante da complexidade de uma doença crônica, como o câncer, que acarreta alterações físicas, psíquicas, sociais e econômicas, sabe-se que há necessidade do planejamento de estratégias que supram as lacunas ocasionadas por essa doença. Desse modo, o sistema de saúde precisa continuar investindo em ações para o controle dessa enfermidade, atuando na prevenção e detecção precoce. ${ }^{2}$

Nesse pensar, não basta apenas desenvolver tecnologias cada vez mais eficientes no tratamento do câncer, sem atentar para a desigualdade social que permeia a sociedade. Para isso, é preciso proporcionar à população acesso facilitado aos recursos e procedimentos disponíveis no sistema de saúde. ${ }^{3}$

Deste modo, reforça-se à necessidade de acompanhar e monitorar as desigualdades sociais existentes, com a intenção de avaliar as demandas não atendidas e 


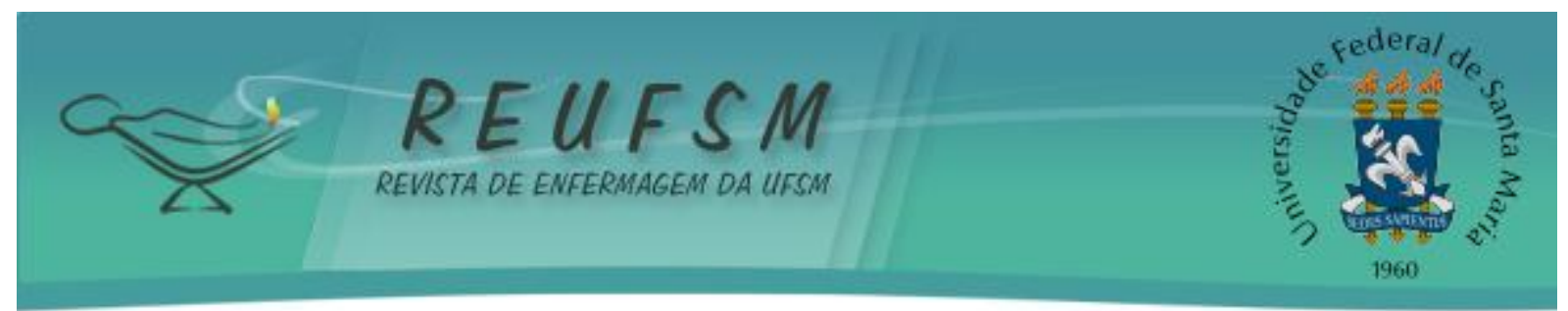

investir na tentativa de desenvolver intervenções eficazes, buscando a equidade no sistema de saúde. ${ }^{4}$

Frente à contextualização do câncer no Brasil e no mundo, pensa-se na relevância do estudo para os profissionais da saúde, pois a partir dessa investigação será possível implantar ações específicas para atender as mulheres nessa condição, além de desenvolver estratégias de promoção da saúde e de prevenção do câncer direcionada a população feminina.

Com base no exposto, a questão norteadora que impulsionou este estudo foi: quais as características das mulheres em tratamento quimioterápico de um serviço de oncologia situado no município de Pelotas, Rio Grande do Sul, Brasil? Assim, tem-se como objetivo descrever o perfil das mulheres em tratamento quimioterápico em um serviço de oncologia de um hospital de ensino em Pelotas, Rio Grande do Sul, Brasil.

\section{MÉTODO}

Trata-se de um estudo descritivo, com abordagem quantitativa, a partir da pesquisa "Os clientes oncológicos e suas famílias e os sistemas de cuidados nas condições crônicas", realizada em um serviço de oncologia de um hospital de ensino no município de Pelotas, Rio Grande do Sul, Brasil. O período de coleta de dados foi compreendido entre os meses de março e junho de 2010.

A amostra do estudo foi constituída por conveniência, resultando em 221 pacientes oncológicos em tratamento quimioterápico, sendo que destes, 117 eram mulheres. As participantes da pesquisa assinaram o Termo de Consentimento Livre e Esclarecido, após serem informadas quanto aos objetivos do estudo.

Os critérios de inclusão consistiram em: ter idade igual ou superior a 18 anos; estar ciente do diagnóstico de câncer e do tratamento; ser capaz de comunicar-se verbalmente para responder aos questionamentos durante a coleta de dados.

As variáveis para este estudo foram selecionadas a partir do instrumento de pesquisa, o qual consistiu em um questionário estruturado. Utilizaram-se as questões relacionadas às características sociodemográficas, econômicas, comorbidades, fatores de risco autorreferidos, número de gestações, realização de exames preventivos e tipo de câncer.

Realizou-se a análise dos dados no programa Stata 9.1 por meio do Stat Transfer, a partir de um banco de dados elaborado no programa Epi-info versão 6.04, o qual sofreu dupla digitação, a fim de corrigir possíveis incoerências. Para a análise estatística, foi realizada a distribuição da frequência das variáveis, sendo apresentada em tabelas.

Antes de proceder com o início da coleta de dados, realizou-se o pré-teste do instrumento em uma população que não foi incluída no estudo. 0 controle de qualidade dos dados ocorreu em todas as etapas da coleta, por meio da checagem de cada instrumento e da revisão dos questionários por parte dos supervisores, para detecção e correção de possíveis inconsistências. A reaplicação do questionário reduzido foi efetivada por meio de contatos telefônicos a 10,0\% dos participantes.

Este estudo respeitou as diretrizes sobre pesquisa com seres humanos, norteadas pela resolução 196/96 do Conselho Nacional de Saúde. ${ }^{5}$ Obteve aprovação pelo Comitê de Ética da Universidade Católica de Pelotas, recebendo parecer favorável sob o número 2008/23 e registro no Conselho Coordenador do Ensino, da Pesquisa e da Extensão (COCEPE) sob o número 4.04.01.015.

\section{RESULTADOS}

A população feminina (Tabela 1 ) encontrou-se predominantemente na faixa etária compreendida entre os 61 anos ou mais, representando 35,0\% (n=41). Em relação à cor da 


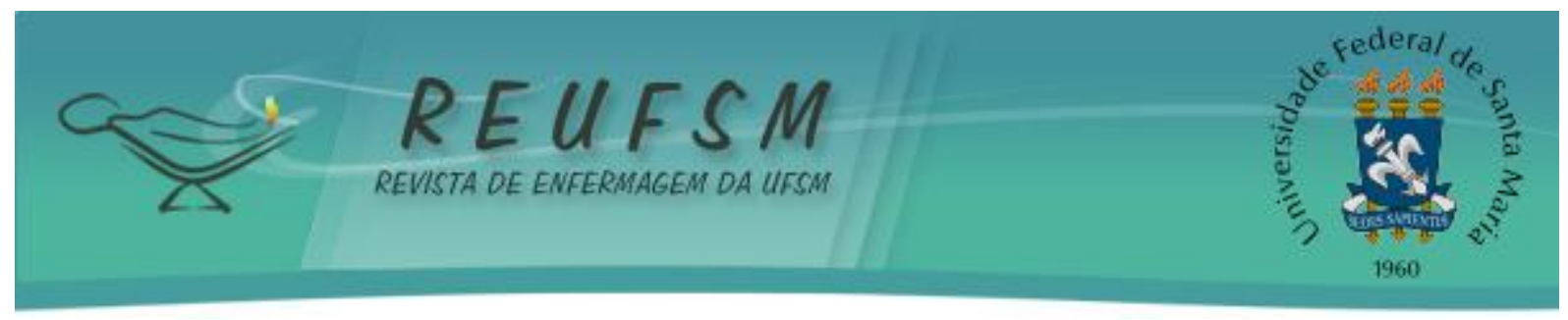

pele, 78,6\% $(n=92)$ das pacientes eram brancas. Quanto à escolaridade, verificou-se que $41,0 \%(n=48)$ das entrevistadas tinham entre cinco e oito anos de estudo. Em relação à religiosidade, 94,0\% ( $n=110)$ possuíam algum tipo de crença religiosa. No que se refere à situação conjugal, 53,8\% (n=63) não apresentavam companheiro. Quanto à procedência, $70,1 \%(n=82)$ eram da zona urbana, sendo a maior parte das participantes do estudo do município de Pelotas, 59,0\% ( $n=69)$. Sobre os dados econômicos, 67,5\% ( $n=77)$ das mulheres referiram ter como renda individual de um a dois salários mínimos.

Tabela 1 - Distribuição da população de mulheres com câncer em tratamento quimioterápico, segundo características sociodemográficas. Pelotas/RS, UFPel, 2010

\begin{tabular}{|c|c|c|}
\hline Sociodemográficas e econômicas & $\mathrm{N}(\mathbf{1 1 7})$ & (\%) \\
\hline \multicolumn{3}{|l|}{ Faixa Etária } \\
\hline $20-30$ anos & 03 & 2,6 \\
\hline $31-40$ anos & 13 & 11,1 \\
\hline $41-50$ anos & 21 & 18,0 \\
\hline $51-60$ anos & 39 & 33,3 \\
\hline 61 anos ou mais & 41 & 35,0 \\
\hline \multicolumn{3}{|l|}{ Cor da pele } \\
\hline Branca & 92 & 78,6 \\
\hline Parda & 14 & 12,0 \\
\hline Preta & 09 & 7,7 \\
\hline Amarela & 02 & 1,7 \\
\hline \multicolumn{3}{|l|}{ Escolaridade } \\
\hline Sem escolaridade & 13 & 11,1 \\
\hline 01 a 04 anos & 33 & 28,2 \\
\hline 05 a 08 anos & 48 & 41,0 \\
\hline 09 a 12 anos & 18 & 15,4 \\
\hline 13 anos ou mais & 05 & 4,3 \\
\hline \multicolumn{3}{|l|}{ Religião } \\
\hline Possui & 110 & 94,0 \\
\hline Não possui & 07 & 6,0 \\
\hline \multicolumn{3}{|l|}{ Situação conjugal } \\
\hline Sem companheiro & 63 & 53,8 \\
\hline Com companheiro & 54 & 46,2 \\
\hline \multicolumn{3}{|l|}{ Procedência } \\
\hline Urbana & 82 & 70,1 \\
\hline \multirow{2}{*}{\multicolumn{3}{|c|}{ Município }} \\
\hline & & \\
\hline Pelotas & 69 & 59,0 \\
\hline Outros municípios da região* & 26 & 22,3 \\
\hline Bagé & 10 & 8,5 \\
\hline São Lourenço do Sul & 06 & 5,1 \\
\hline Capão do Leão & 06 & 5,1 \\
\hline Renda Individual** & $N(114)$ & (\%) \\
\hline Menos de um salário & 28 & 24,6 \\
\hline 1 a 2 salários & 77 & 67,5 \\
\hline 3 a 4 salários & 08 & 7,0 \\
\hline 5 a mais salários & 01 & 0,9 \\
\hline
\end{tabular}

Fonte: Banco de dados da pesquisa "Os clientes oncológicos e suas famílias e os sistemas de cuidado nas condições crônicas", Pelotas-RS, 2010

* Compreendem os municípios da $3^{\text {a } C o o r d e n a d o r i a ~ R e g i o n a l ~ d e ~ S a u ́ d e ~}$

**Variável com o maior número de ignorados $(n=114)$ 


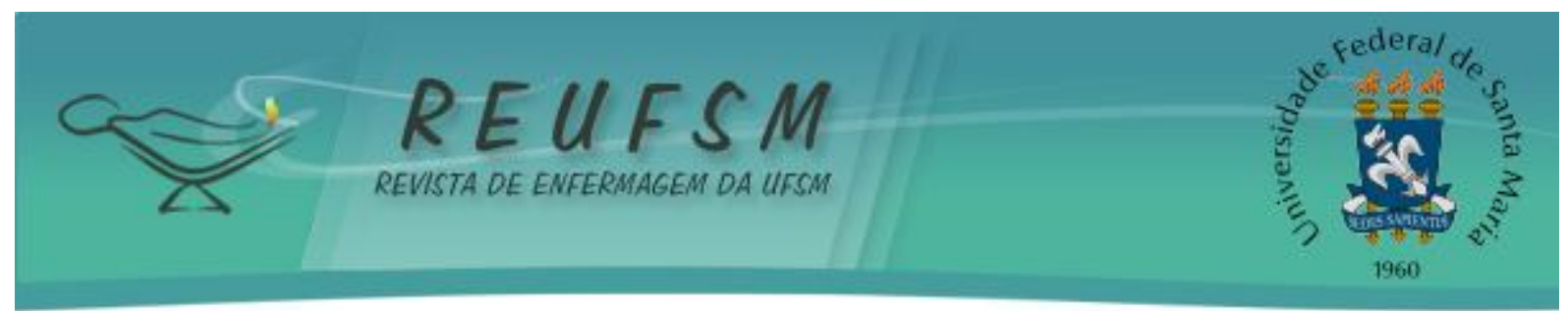

Observa-se que a hipertensão, com 39,3\% $(n=46)$ foi a comorbidade mais relatada pelas participantes do estudo, seguida da doença cardíaca e do diabetes, aos quais apresentaram taxas iguais, 13,7\% ( $\mathrm{n=16}$ ), conforme apresentado na Tabela 2.

Tabela 2 - Distribuição da população de mulheres com câncer em tratamento quimioterápico, de acordo com as comorbidades autorreferidas. Pelotas/RS, UFPel, 2010

\begin{tabular}{lcc}
\hline \multicolumn{1}{c}{ Comorbidades } & $\mathbf{N ( 1 1 7 )}$ & $\mathbf{( \% )}$ \\
\hline Doença cardíaca & 16 & 13,7 \\
Diabetes & 16 & 13,7 \\
Hipertensão & 46 & 39,3 \\
Neurológico & 08 & 6,8 \\
Cardiocirculatório & 07 & 6,0 \\
Esquelético & 07 & 6,0 \\
Respiratório & 05 & 4,3 \\
Gastrointestinal & 03 & 2,6 \\
Geniturinário & 01 & 0,8 \\
\hline
\end{tabular}

Fonte: Banco de dados da pesquisa "Os clientes oncológicos e suas famílias e os sistemas de cuidado nas condições crônicas”, Pelotas-RS, 2010

A Tabela 3 ilustra as variáveis relativas aos fatores de risco autorreferidos pelas participantes do estudo. Em relação à história familiar de câncer, 72,8\% $(n=83)$ afirmaram ter algum integrante da família que já apresentou a doença. Quanto ao tabagismo, 8,6\% $(n=10)$ relataram o uso. No que diz respeito à ingestão de bebidas alcoólicas, $29,9 \%(n=35)$ referiram ingerir ou já ter ingerido. Referente ao hábito de ingerir chimarrão, 81,2\% ( $n=95)$ das mulheres o mencionaram. Já a temperatura da água do chimarrão, $66,3 \% \quad(n=63)$ relataram que a temperatura era quente.

Tabela 3 - Distribuição da população de mulheres com câncer em tratamento quimioterápico, de acordo com fatores de risco autorreferidos, Pelotas/RS, UFPel, 2010

\begin{tabular}{lcc}
\hline \multicolumn{1}{c}{ Fatores de risco referido } & $\mathbf{N}(\mathbf{1 1 7})$ & $\mathbf{( \% )}$ \\
\hline Familiares com câncer & 83 & 72,8 \\
Tabagista & 10 & 8,6 \\
& 46 & 39,3 \\
Ingestão de bebida alcoólica & 35 & 29,9 \\
Hábito de chimarrão & 95 & 81,2 \\
Temperatura da água do chimarrão* & $\mathbf{N}(95)$ & $(\%)$ \\
Quente & 63 & 66,3 \\
Morna & 30 & 31,6 \\
Fervente & 02 & 2,1 \\
\hline
\end{tabular}

Fonte: Banco de dados da pesquisa "Os clientes oncológicos e suas famílias e os sistemas de cuidado nas condições crônicas", Pelotas-RS, 2010

*Variável com o maior número de ignorados $(n=95)$

Ainda, neste estudo (Tabela 4), constata-se que a maioria das mulheres teve de uma a três gestações, 47,9\% $(n=56)$. Em relação ao exame citopatológico, $97,4 \%(n=114)$ disseram que realizaram o exame preventivo, sendo que $47,2 \%(n=50)$ o fizeram no período de um a quatro anos.

No que diz respeito à mamografia, $82,1 \%(n=96)$ das participantes afirmaram ter realizado o exame, sendo que $52,1 \%(n=50)$ fizeram em um intervalo menor do que um ano. A realização de ultrassonografia foi negada por $60,7 \%(n=71)$, sendo que, das participantes que a fizeram, $66,7 \%(n=30)$ a realizaram em um período menor do que um 


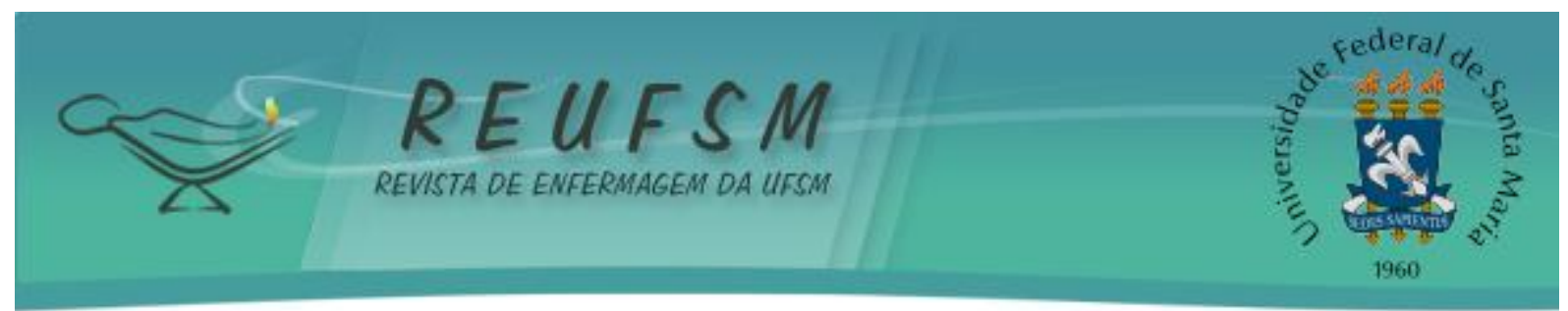

ano. Quanto ao autoexame das mamas, $67,5 \%(n=79)$ alegaram realizar o procedimento. $E$ o tipo de câncer predominante na população do estudo foi o da mama, 41,0\%.

Tabela 4 - Distribuição da população de mulheres com câncer em tratamento quimioterápico, referente a número de gestações, realização de exames preventivos e tipo de câncer. Pelotas/RS, UFPel, 2010

Gestações, exames preventivos e tip
Nenhuma
01 a 03
04 a 06
07 a 09

Não

Tempo de realização do último pré-câncer

Menos de 1 ano

1 a 4 anos

5 a 10 anos

11 a 20 anos

Mamografia

$\operatorname{Sim}$

Não

Tempo de realização da última mamografia

Menos de 1 ano

$N(117)$

(\%)

$\begin{array}{cc}16 & 13,7 \\ 56 & 47,9 \\ 37 & 31,6 \\ 08 & 6,8\end{array}$

1 a 4 anos

5 a 10 anos

$\mathrm{N}(117)$

114

(\%)

03

$\mathrm{N}(106)$

97,4

$44 \quad 41,5$

$50 \quad 47,2$

$09 \quad 8,5$

$03 \quad 2,8$

11 a 20 anos

Ultrassonografia

Não

Sim

Tempo de realização da última ultrassonografia*

96

21

82,1

17,9

$\mathrm{N}(96) \quad$ (\%)

$50 \quad 52,1$

$41 \quad 42,7$

$04 \quad 4,2$

$01 \quad 1,0$

Menos de 1 ano

$71 \quad 60,7$

$46 \quad 39,3$

1 a 4 anos

5 a 10 anos

N(45)

(\%)

$30 \quad 66,7$

$11 \quad 24,4$

11 a 20 anos

03

24,4
6,7

01

2,2

Sim

Autoexame

Não

Tipo de câncer

Mama

Intestino

Pulmão

Outros tipos de câncer

$79 \quad 67,5$

$38 \quad 32,5$

Colo uterino

N(117)

32,5

$48 \quad 41,0$

$22 \quad 18,8$

$15 \quad 12,8$

Ovário

12

10,3

$10 \quad 8,5$

$05 \quad 4,3$

Sistema hematopoiético

05

4,3

Fonte: Banco de dados da pesquisa "Os clientes oncológicos e suas famílias e os sistemas de cuidado nas condições crônicas”, Pelotas-RS, 2010

* Variável com o maior número de ignorados $(\mathrm{n}=45)$ 


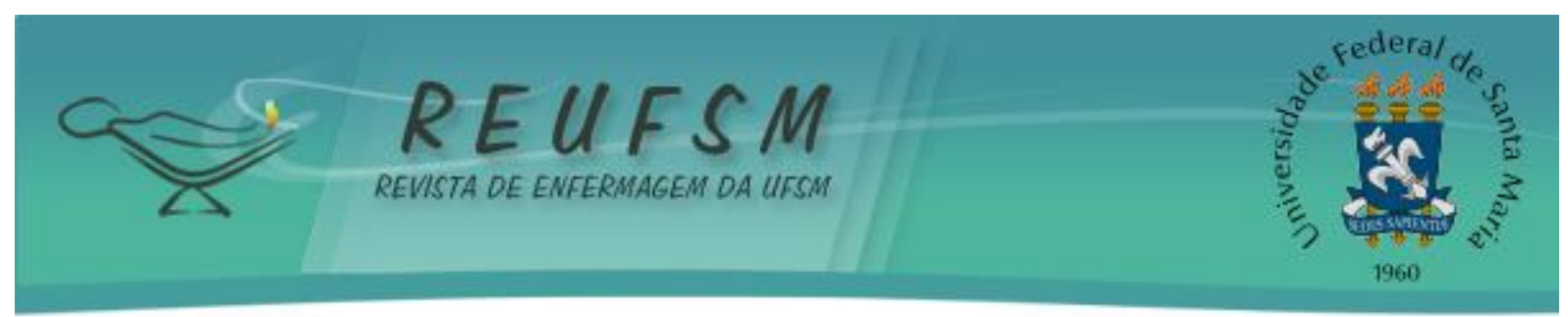

\section{DISCUSSÃO}

A partir da análise do presente estudo, evidenciou-se que a maioria das mulheres estava na faixa etária dos 61 anos ou mais, representando 35,0\% ( $n=41)$. Fator esse, semelhante aos resultados de uma investigação realizada na Suíça. ${ }^{6}$

Em decorrência do aumento da expectativa de vida, há uma expansão da população idosa, consequentemente, uma maior exposição a fatores de risco para o desenvolvimento de doenças crônico-degenerativas, tornando-se mais frequentes as complicações por esses agravos. O câncer consiste em uma dessas doenças que influencia na morbimortalidade da população nessa faixa etária, mundialmente. ${ }^{7}$

A cor da pele branca foi referida em 78,6\% (n=92). Destaca-se que no Rio Grande do Sul, a maior parte da população possui essa característica, o que pode ter influenciado neste resultado. Dados de uma pesquisa realizada em Santa Catarina, na qual avaliaram a sobrevida e os fatores prognósticos de mulheres com câncer da mama, identificou que a raça/cor branca predominou, 94,1\% em um total de 1.002 participantes, estando a cor da pele associada à melhor sobrevida em comparação com as mulheres negras, pardas, amarelas e indígenas. ${ }^{8}$

Em se tratando de escolaridade, essa pode influenciar em diversos aspectos relacionados à saúde da população, sendo que a maior parte das mulheres referiu de cinco a oito anos. Esse dado assemelha-se a uma pesquisa desenvolvida no Sul do Brasil, com sobreviventes ao câncer, em que 38,6\% apresentaram esse nível de escolaridade, equivalendo à maior frequência. ${ }^{9}$

0 presente estudo está de acordo com outra pesquisa realizada ${ }^{10}$, no qual o predomínio de escolaridade foi de cinco a oito anos, equivalendo a 41,0\% $(n=48)$. Entretanto, uma parcela significativa apresentou de zero a quatro anos, o que demonstra a baixa escolaridade de algumas participantes.

Majoritariamente, as participantes referiram possuir algum tipo de crença religiosa, sendo as religiões, católica e evangélica, as mais citadas. Esse fato foi ressaltado positivamente em um estudo ${ }^{11}$ que entrevistou 20 idosos com câncer, tendo encontrado que a fé auxilia a pessoa a se preparar e a se fortalecer para batalhar contra as adversidades. Ainda, reforçam que a fé resulta em esperança, equilíbrio, fortalecimento e aceitação da doença.

Mais de $50,0 \%$ das mulheres referiu não apresentar companheiro. Variável semelhante ao resultado de uma investigação realizada no Sul do Brasil que apontou que $52,6 \%$ dos sobreviventes ao câncer, apresentaram como estado civil, ser casado(a) ou ter companheiro(a). ${ }^{9}$

A inexistência de serviços de saúde específicos à atenção oncológica no município de origem das pacientes, demandou a necessidade de deslocamento para outras cidades que sejam referência em tratamento de alta complexidade. Com isso, destaca-se que parte da população deste estudo, teve como procedência outras regiões do sul do Rio Grande do Sul, embora 59,0\% ( $n=69)$ das participantes eram do município de Pelotas.

Em uma pesquisa foi evidenciado que, de um total de 310 pacientes, 98 não tinham serviço de assistência oncológica no município de origem. Os autores, ainda destacaram que a disponibilidade e a qualidade do cuidado provido, os métodos de detecção precoce e a facilidade no acesso aos serviços de saúde, influenciam diretamente no desenvolver da doença. ${ }^{12}$

Em relação à renda, em uma investigação realizada, foi descrito que 60,6\% das 102 participantes tinham renda individual de um salário mínimo. ${ }^{10}$ Dado que corrobora com o presente estudo, em que $67,5 \%(n=77)$ tinham renda individual de um a dois salários mínimos. Autores pontuam que mulheres em condições socioeconômicas desfavoráveis são mais vulneráveis ao surgimento de doenças, como o câncer, principalmente em estágio avançado, devido ao diagnóstico tardio em virtude da demora na realização de alguns exames. ${ }^{13}$ 


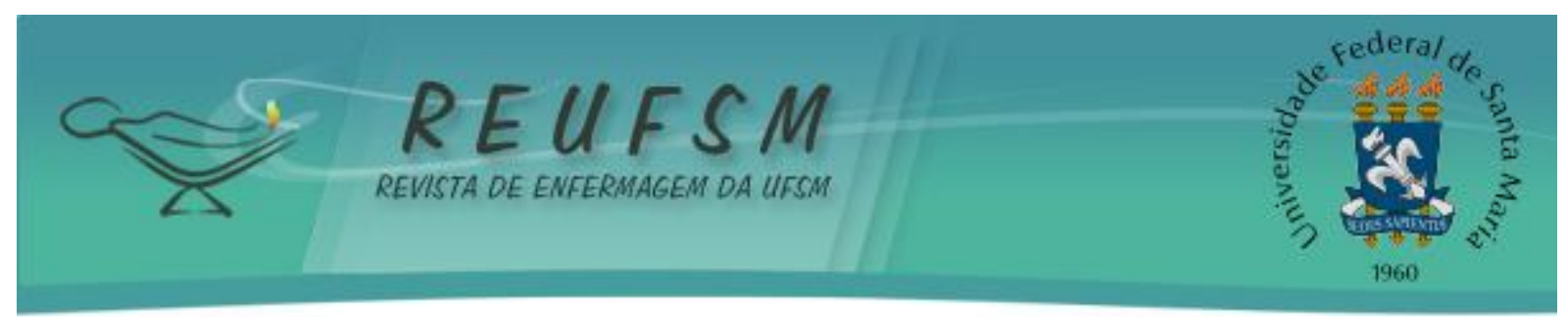

Em uma pesquisa ${ }^{14}, 23,8 \%$ das mulheres responderam ser hipertensas e $4,4 \%$ referiram ser diabéticas, de um total de 1.239 participantes. Esses dados encontrados apresentam-se em proporção diferente ao que foi encontrado no presente estudo, em que $39,3 \%(n=46)$ das mulheres referiram ser hipertensas e 13,7\% $(n=16)$ diabéticas, o que pode estar relacionado com a diferença do número de participantes entrevistadas e às diferenças das populações estudadas, uma vez que as pessoas entrevistadas na pesquisa realizada $^{14}$ não estavam dentro de um serviço de saúde. Já em outra investigação ${ }^{10}$, na qual participaram 102 mulheres, 64,7\% referiram ser hipertensas e 18,8\% diabéticas.

A hereditariedade consiste em um dos fatores não modificáveis para o aparecimento do câncer. De acordo com o Instituto Nacional de Câncer, a história familiar influencia no surgimento dos cânceres da mama, pulmão, colorretal e esôfago. ${ }^{2}$ Fator esse, afirmado por $72,8 \%(n=83)$ das participantes.

Referente aos fatores modificáveis, para o surgimento do câncer, foi relatada a existência de diversos. No que se refere ao tabagismo, esse era um hábito predominantemente masculino na sociedade moderna. Entretanto, após o movimento social do feminismo e a inserção das mulheres no meio capitalista, fumar tornou-se um costume, também delas. Além disso, o uso do tabaco está diretamente relacionado com as classes sociais, atingindo principalmente populações com baixa renda e menor escolaridade. ${ }^{15}$

Em uma pesquisa ${ }^{14}$, por meio de ligações telefônicas para domicílios de moradores da cidade de Goiânia, sobre fatores de risco para doenças crônicas, de um total de 1.239 mulheres, foi encontrado que $8,7 \%$ eram tabagistas e $22,0 \%$ referiram consumir bebida alcoólica. Fato esse condizente com o presente estudo, em que 8,6\% $(n=10)$ das mulheres relataram o uso do tabaco e $29,9 \%(n=35)$ ingerir ou já ter ingerido bebida alcoólica.

De acordo com uma investigação ${ }^{15}$ realizada, o Rio Grande do Sul é um dos estados com maior prevalência de tabagismo, tendo elevada incidência de neoplasias associadas com o tabaco: cavidade oral, esôfago e pulmão.

Outro hábito da população gaúcha é a ingesta de chimarrão, o que foi mencionado por $81,2 \%(n=95)$ das participantes do estudo. A temperatura da água predominantemente utilizada foi a quente, entretanto, não existe um consenso na literatura em relação ao consumo do chimarrão e a carcinogênese bucal. Salienta-se que em uma pesquisa realizada, a frequência das neoplasias do trato digestivo superior foi mais elevada em regiões em que o consumo de chimarrão é maior. Neste sentido, as autoras acreditam em uma associação desse hábito com a presença da doença, sobretudo pela injúria térmica, ainda mais quando existem outros fatores de risco, como o tabaco e o consumo de bebida alcoólica. ${ }^{16}$

Quanto ao número de gestações, majoritariamente, a amostra de 47,9\% $(n=56)$, mencionou ter de uma a três. Esse dado diverge de uma investigação ${ }^{17}$, em que foi descrito que a nuliparidade e o reduzido número de gestações são considerados fatores importantes para o desenvolvimento do câncer da mama.

No estudo em questão, $97,4 \%(n=114)$ das mulheres relatou realizar o exame citopatológico de colo uterino, podendo ser um dos fatores que influenciou na baixa frequência desta neoplasia. Dados semelhantes foram encontrados em uma pesquisa ${ }^{18} \mathrm{com}$ 885 mulheres no município de Guarapuava-Paraná, em que aproximadamente $80,0 \%$ das entrevistadas realizaram esse exame. Além do mais, a associação da faixa etária das mulheres com a realização do exame foi estatisticamente significativa, havendo maior participação daquelas com idade entre 29 e 58 anos.

Em uma investigação, foi identificada que a maioria das mulheres entrevistadas tinha conhecimento em relação ao objetivo e a importância do citopatológico de colo uterino, além de questões relacionadas à patologia, como o seu início assintomático. ${ }^{19}$

No presente estudo, $17,9 \%(n=21)$ das mulheres referiram não ter realizado a mamografia. Entretanto, o motivo não foi investigado. Uma das hipóteses pode estar 


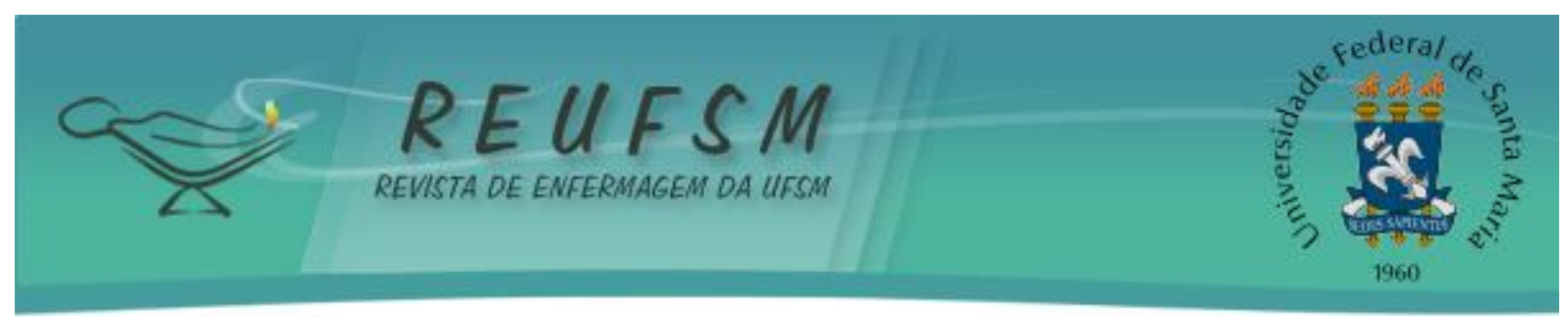

relacionada à população de mulheres que não estavam compreendidas na faixa etária de risco para realização desse exame.

Em outra pesquisa ${ }^{18}$, apenas $24,0 \%$ das mulheres haviam realizado mamografia. Os autores associaram esse fato, com o menor nível de formação escolar e a baixa renda. Ainda, sugerem que este exame é de difícil acesso à maior parte da população feminina, principalmente a desfavorecida socioeconomicamente.

Embora o número de mulheres que não realizaram o exame mamográfico tenha sido relativamente baixo, destaca-se a proporção de mulheres que apresentaram a neoplasia de mama neste estudo, o que evidencia a necessidade de estratégias que ampliem as ações referentes ao rastreamento precoce do câncer da mama.

Quando questionadas sobre a ultrassonografia, a maioria das participantes do estudo, $60,7 \%$ ( $n=71)$, negou sua realização. Todavia, uma grande parcela das mulheres que realizaram o exame foi em menos de um ano, o que pode estar relacionado com o fato do acompanhamento do quadro clínico, em consequência da presença do câncer da mama, neoplasia mais incidente.

Contudo, sabe-se que a população que utiliza o sistema público de saúde ainda enfrenta percalços na realização de exames como a ultrassonografia, aguardando por longos períodos de tempo para serem contempladas. Isso pode influenciar negativamente no processo de desenvolvimento de doenças como as neoplasias, a partir do retardamento no seu diagnóstico.

Quanto ao autoexame das mamas, $67,5 \%(n=79)$ alegaram realizar o procedimento. Esse não deve ser um método isolado de rastreamento, pois pode influenciar negativamente no diagnóstico do câncer nesta localização. Contudo, é favorável para o autocuidado das mamas e redução dos casos avançados em locais desprovidos de exames de imagem. ${ }^{20}$ Destacase que o Instituto Nacional de Câncer pontua que o autoexame das mamas não substitui a avaliação dos profissionais da saúde capacitados para desenvolver essa atividade. ${ }^{21}$

A partir da análise dos dados, o câncer da mama foi o mais frequente, atingindo $41,0 \%(n=48)$ das participantes. 0 que está de acordo com uma investigação ${ }^{22}$ que traçou o perfil de 321 mulheres com câncer ginecológico em tratamento quimioterápico, o qual também encontrou o câncer da mama como o mais incidente.

Já uma pesquisa realizada em um Centro Regional de Câncer, na cidade da Índia, durante o período de 2009 a 2011, constatou que o câncer de esôfago foi o mais comum entre as mulheres, em seguida, o câncer da mama. Tais dados encontrados, levam a observar que a incidência no sítio da doença pode variar entre os países. ${ }^{23}$

\section{CONCLUSÃO}

Os resultados desta pesquisa demonstram um grupo composto por mulheres que podem ser consideradas de risco para o desenvolvimento de diversos tipos de câncer, pois possuíam idade avançada, com escolaridade e renda baixa. Todavia, o reduzido tamanho amostral foi um dos fatores limitantes para o estudo, e o método descritivo, impossibilitou que correlações fossem estabelecidas.

É importante destacar que alguns fatores de risco modificáveis para o câncer foram referidos pelas participantes do estudo. Assim, pensa-se na importância do conhecimento e da detecção desses hábitos, a fim de que os profissionais da saúde possam implementar ações que visem sua modificação.

No que diz respeito à neoplasia mais frequente, essa foi a da mama, desse modo, enfatiza-se a necessidade de investigações que busquem conhecer melhor a causalidade da alta prevalência dessa neoplasia. Vale destacar que, no Brasil, são desenvolvidas atividades para o controle dos cânceres prevalentes na população feminina, em que se encontra o da 


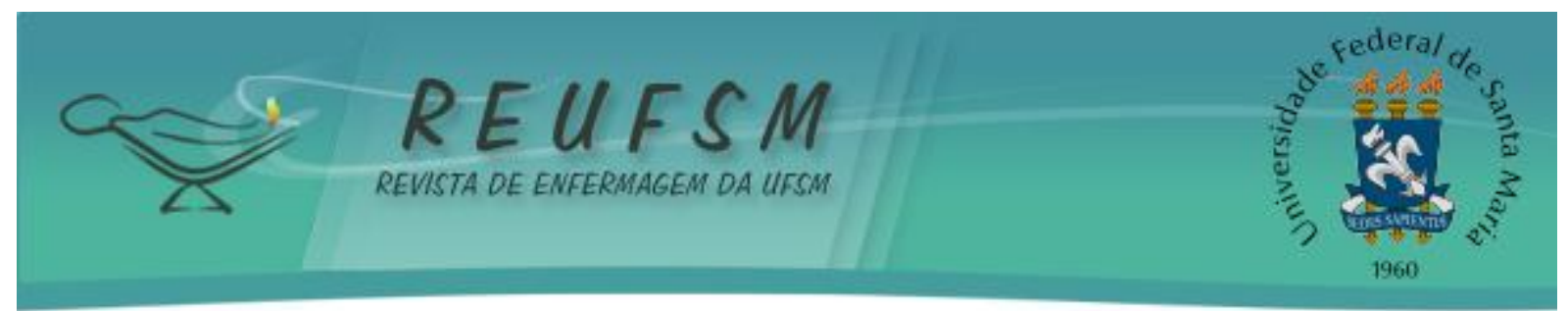

mama. Entretanto, pontua-se que esse tipo de câncer permanece sendo o que mais acomete as mulheres.

A partir deste estudo, acredita-se na relevância de se conhecer o perfil da população atendida, com o propósito de desenvolver estratégias que estimulem a promoção da saúde, prevenção de doenças e reabilitação. Além disso, tem-se a intenção de estimular a produção de outras pesquisas que possam relacionar a presença do câncer com fatores de risco nesta população, assim como sua prevenção, além de qualificar a assistência prestada.

\section{REFERÊNCIAS}

1. World Health Organization (WHO). Fact Sheet $\mathrm{N}^{\circ}$ 297. Genebra: WHO/CHP [Internet] 2011. [acesso em 2011 jun 7]. Disponível em: http://www.who.int/mediacentre/factsheets/fs297/en/index.html.

2. Ministério da Saúde (BR). Instituto Nacional de Câncer (INCA). Estimativa 2012: incidência de câncer no Brasil [Internet]. Rio de Janeiro (RJ): INCA; 2011 [acesso em 2013 out 25]. Disponível em: http://www.inca.gov.br/estimativa/2012/estimativa20122111.pdf.

3. Nogueira ACC, Silva LB. Saúde, gênero e serviço social: contribuições sobre o câncer e saúde da mulher. Vértices [Internet]. 2009 [acesso em 2013 out 25];11(1/3):7-17. Disponível em: http://www.essentiaeditora.iff.edu.br/index.php/vertices/article/download/8/3\%E2\%80\%8E.

4. Louvison MCP, Lebrão ML, Duarte YAO, Laurenti R. Desigualdades nas condições de saúde e no uso dos serviços entre as pessoas idosas do município de São Paulo: uma análise de gênero e renda. Saúde Coletiva [Internet]. 2008 [acesso em 2013 out 25];5(24):189-94. Disponível em: http://www.redalyc.org/pdf/842/84252407.pdf.

5. Ministério da Saúde (BR). Conselho Nacional de Saúde. Resolução CNS nº 196, de 10 de outubro de 1996. Diretrizes e normas regulamentadoras de pesquisa envolvendo seres humanos [Internet]. 1996 [acesso em 2013 out 25]. Disponível em: http://conselho.saude.gov.br/web_comissoes/conep/aquivos/resolucoes/23_out_versao_fi nal_196_ENCEP2012.pdf.

6. Schmidlina K, Spoerria A, Eggera M, Zwahlena M, Stuckc A, Clough-Gorra KM. Cancer, a disease of aging (part 2) - risk factors for older adult cancer mortality in Switzerland 19912008. Swiss med wkly [Internet]. 2012 [acesso em 2013 out 25];142:1-10. Disponível em: http://www.smw.ch/content/smw-2012-13607/.

7. Basílio DV, Mattos IE. Câncer em mulheres idosas das regiões Sul e Sudeste do Brasil: evolução da mortalidade no período 1980-2005. Rev Bras Epidemiol [Internet]. 2008 [acesso em 2013 out 25];11(2):204-14. Disponível em: http: / / www.scielo.br/scielo.php?script=sci_arttext\&pid=S1415-

$790 \times 2008000200003 \& \operatorname{lng}=e n \& n r m=i s o \& t \operatorname{lng}=p t$.

8. Schneider IJC, d'Orsi E. Sobrevida em cinco anos e fatores prognósticos em mulheres com câncer da mama em Santa Catarina, Brasil. Cad Saude Pública [Internet]. 2009 [acesso em 2013 out 25];25(6):1285-96. Disponível em: http://www.scielo.br/scielo.php?script=sci_arttext\&pid=S0102-311X2009000600011.

9. Andrade FP, Muniz RM, Lange C, Guanilo MEE. Perfil sociodemográfico e econômico dos sobreviventes ao câncer segundo o grau de resiliência. Texto \& Contexto Enferm [Internet]. 2013 [acesso em 2013 out 25];22(2):476-84. Disponível em: http://www.scielo.br/pdf/tce/v22n2/v22n2a25.pdf. 


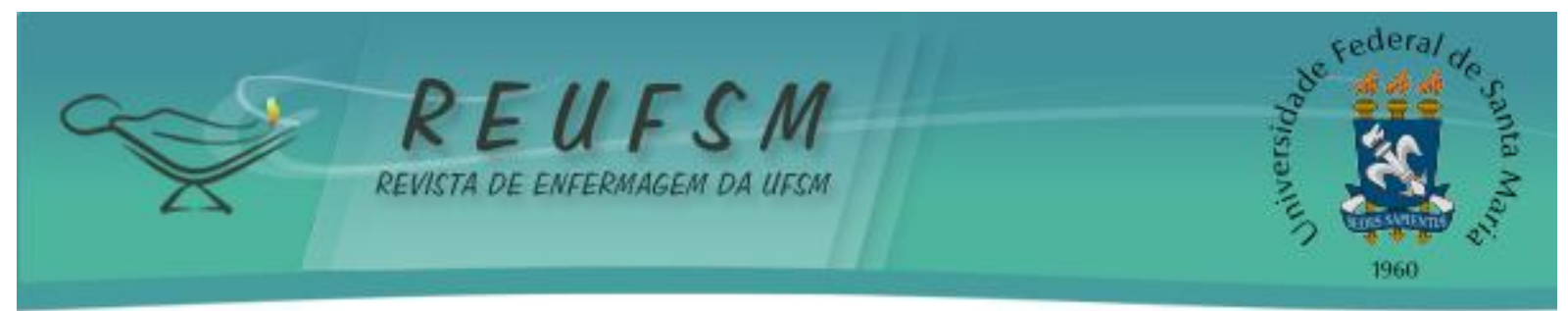

10. Assis LS, Stipp MAC, Leite JL, Cunha NM. A atenção da enfermeira à saúde cardiovascular de mulheres hipertensas. Esc Anna Nery Rev Enferm [Internet]. 2009 [acesso em 2013 out 25];13(2):265-70. Disponível em: http: / /www.eean.ufrj.br/revista_enf/20092/artigo\%203.pdf.

11. Teixeira JJV, Lefèvre F. Significado da intervenção médica e da fé religiosa para o paciente idoso com câncer. Cienc Saúde Coletiva [Internet]. 2008 [acesso em 2013 out 25];13(4):1247-56. Disponível em: http://www.scielo.br/scielo.php?script=sci_arttext\&pid=S1413$81232008000400021 \& \operatorname{lng}=$ pt\&nr.

12. Brito C, Portela MC, Vasconcellos MTL. Sobrevida de mulheres tratadas por câncer da mama no estado do Rio de Janeiro. Rev Saude Publica [Internet]. 2009 [acesso em 2013 out 25];43(3):481-9. Disponível em: http://www.scielo.br/pdf/rsp/v43n3/202.pdf.

13. Mendonça VG, Lorenzato FRB, Mendonça JG, Menezes TC, Guimarães MJB. Mortalidade por câncer do colo do útero: características sociodemográficas das mulheres residentes na cidade de Recife, Pernambuco. Rev Bras Ginecol Obstet [Internet]. 2008 [acesso em 2013 out 25];30(5):248-55. Disponível em: http://www.scielo.br/scielo.php?script=sci_arttext\&pid=S010072032008000500007.

14. Peixoto MRG, Monego ET, Alexandre VP, Souza RGM, Moura EC. Monitoramento por entrevistas telefônicas de fatores de risco para doenças crônicas: experiência de Goiânia, Goiás, Brasil. Cad Saude Publica [Internet]. 2008 [acesso em 2013 out 25];24(6):1323-33. Disponível em: http://www.scielo.br/pdf/csp/v24n6/13.pdf.

15. Wunsch Filho V, Mirra AP, López RVM, Antunes LF. Tabagismo e câncer no Brasil: evidências e perspectivas. Rev Bras Epidemiol [Internet]. 2010 [acesso em 2013 out 25];13(2):175-87. Disponível em: http://www.scielo.br/scielo. php?pid=S1415790X2010000200001\&script=sci_arttext.

16. Sehnem S, Veltrini VC. O chimarrão e suas repercussões bucais. Rev Saude Pesquisa [Internet]. 2012 [acesso em 2013 out 25];5(3):447-53. Disponível em: http://www.unicesumar.edu.br/pesquisa/periodicos/index.php/saudpesq/article/viewFile /2062/1800.

17. Matos JC, Pelloso SM, Carvalho MDB. Prevalência de fatores de risco para o câncer da mama no município de Maringá, Paraná. Rev Latinoam Enferm [Internet]. 2010 [acesso em 2013 out 25];18(3):57-64. Disponível em: http://www.scielo.br/pdf/rlae/v18n3/pt_09.pdf.

18. Bim CR, Pelloso SM, Carvalho MDB, Previdelli ITS. Diagnóstico precoce do câncer da mama e colo uterino em mulheres do município de Guarapuava, PR, Brasil. Rev Esc Enferm USP [Internet]. 2010 [acesso em 2013 out 25];44(4):940-6. Disponível em: http://www.scielo.br/pdf/reeusp/v44n4/12.pdf.

19. Wünsch S, Oliveira SG, Garcia RP, Domingues IB. Coleta de citopatológico de colo uterino: saberes e percepções de mulheres que realizam o exame. Rev Enferm UFSM [Internet]. 2011 [acesso em 2013 out 25];1(3):360-8. Disponível em: http://cascavel.ufsm.br/revistas/ojs-2.2.2/index.php/reufsm/article/view/2543/2385.

20. Menke $\mathrm{CH}$, Delazeri GJ. Autoexame ou autoengano? Feminina [Internet]. 2010 [acesso em 2013 out 25];38(1):3-6. Disponível em: http://files.bvs.br/upload/S/01007254/2010/v38n1/a002.pdf.

21. Ministério da Saúde (BR). Instituto Nacional de Câncer (INCA). Câncer da mama [Internet]. [acesso em 2013 jan 24]. Disponível em: http://www.inca.gov.br/conteudo_view.asp?id=336. 


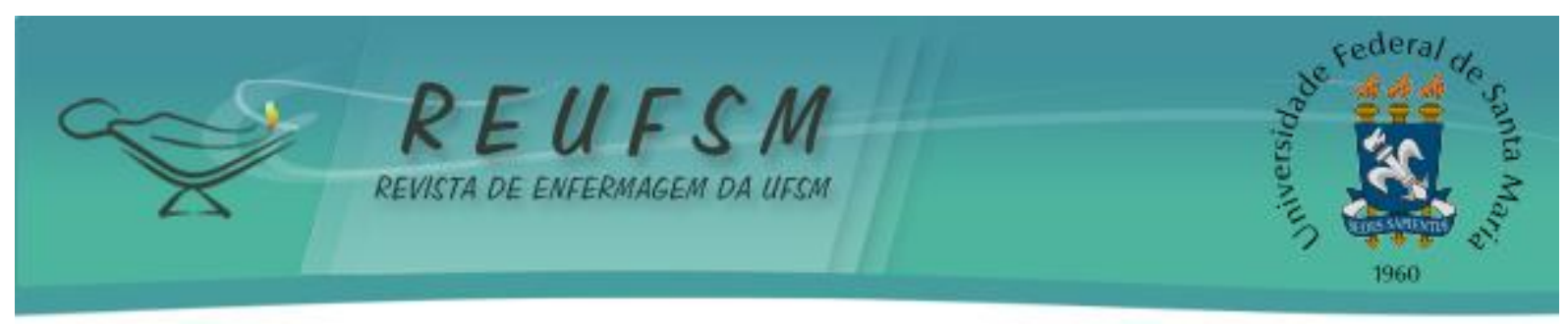

22. Soares EM, Silva SR. Perfil de pacientes com câncer ginecológico em tratamento quimioterápico. Rev Bras Enferm [Internet]. 2010 [acesso em 2013 out 25];63(4):517-22. Disponível em: http://www.scielo.br/pdf/reben/v63n4/03.pdf.

23. Rasool MT, Lone MM, Wani ML, Afroz F, Zaffar S, Haq MM. Cancer in Kashmir, India: Burden and pattern of disease. J Can Res Ther [Internet]. 2012 [acesso em 2013 out 25];8(2):243-6. Disponível em: http://www.cancerjournal.net/article.asp?issn=09731482; year=2012; volume=8; issue=2; spage $=243$; epage $=246$; aulast $=$ Rasool.

Data de recebimento: 29/08/2013

Data de aceite: 30/01/2014

Contato com autor responsável: Aline da Costa Viegas

Endereço postal: Rua: Demétrio Ribeiro № 1115, Fragata, Pelotas, RS, Brasil.

E-mail: alinecviegas@hotmail.com. 